06

\title{
Модифицированная модель Друде-Лоренца, позволяющая учесть топологические характеристики среды
}

\author{
(C) А.И. Маймистов ${ }^{1}$, Е.И. Ляшко ${ }^{2}$ \\ ${ }^{1}$ Национальный исследовательский ядерный университет МИФИ, \\ 115409 Москва, Россия \\ ${ }^{2}$ Московский фризико-технический институт, \\ 141700 Долгопрудный, Московская обл., Россия \\ e-mail: aimaimistov@gmail.com, ostroukhova.ei@gmail.com
}

Поступила в редакцию 08.05.2019 г.

В окончательной редакции 08.05.2019 г.

Принята к публикации 11.06.2019 г.

Модель Друде-Лоренца, которая позволяет описать нелинейный отклик диэлектрической или проводящей среды, может быть приспособлена для описания нелинейных нерезонансных откликов некоторых экзотических сред: топологических изоляторов, полуметалла Вейля или металла Дирака. Представлены обобщенная модель Друде-Лоренца и ее упрощенный вариант, в котором топологические эффекты учитываются в минимальной степени. Как пример применения упрощенной модели дан вывод нелинейной проводимости второго порядка, отвечающей за генерацию второй гармоники и эффект оптического выпрямления. Показано, что отношение топологической проводимости к обычной линейной проводимости содержит константы, пропорциональные постоянной тонкой структуры и градиенту аксионного поля.

Ключевые слова: аксионное поле, связность Берри, кривизна Берри, топологический изолятор, сила Лоренца, нелинейная проводимость, поляризуемость, генерация второй гармоники.

DOI: $10.21883 /$ OS.2019.11.48519.155-19

\section{Введение}

Для описания диэлектрической проницаемости часто используется модель Друде-Лоренца (ДЛ), в которой среда представлена ансамблем гармонических осцилляторов, помещенных в электромагнитное поле [1]. В этой модели предполагается, что на каждую электрически за-

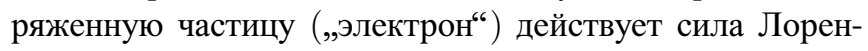
ца, сила трения и сила, удерживающая „электрон“ около положения равновесия. Можно показать, что модель ДЛ в классическом приближении следует из квантово-механической модели заряженной (нерелятивистской) частицы, находящейся в электромагнитном поле и удерживающем потенциале [2]. Полагая удерживающий потенциал гармоническим, можно получить модель гармонического осциллятора с вынуждающей силой Лоренца - обычную модель Друде-Лоренца. Несмотря на недостатки модели ДЛ, она широко используется. Так, в настоящее время электромагнитные свойства плазмонных материалов и метаматериалов часто обсуждаются в рамках этой модели или на основе модели свободных электронов, когда удерживающий потенциал отсутствует (модель Друде) $[3,4]$. Динамика электронов в кристаллах в квазиклассическом приближении описывается как движение волнового пакета под действием электромагнитного поля и эффективного магнитного поля, которое возникает из-за нетривиальных топологических свойств функций Блоха [5-8]. Положение центра волнового пакета и сопряженный импульс удовлетворяют уравнениям Га- мильтона, которые можно (в некотором случае) свести к уравнениям ДЛ.

Если в модели ДЛ учесть негармоничность удерживающего потенциала, то негармоническое движение заряженной частицы в этом потенциале приведет к нелинейным откликам диэлектрика [9-11]. Помимо этого, сила Лоренца делает движение заряженной частицы негармоническим и происходит сложение или вычитание частот действующих гармонических полей. Непараболическая зависимость энергии электрона от квазиимпульса в зоне Бриллюэна также является причиной нелинейного отклика на внешнее электромагнитное поле. Если эффективное магнитное поле (оно известно под названием связности Берри [12]) не равно нулю, то возникает еще одна причина нелинейного отклика среды на действие электромагнитного поля.

В настоящей работе рассмотрены две формулировки обобщенной модели Друде-Лоренца. Влияние диссипации включено в модель феноменологически. Потенциал, удерживающей заряд, можно также добавить в модель, если в том есть необходимость. Основное внимание уделяется топологическим аспектам модели, которые могут проявить себя в нелинейной оптике. Исходя из присущего топологическому изолятору магнитоэлектрического эффекта будет определена сила, действующая на изолятор. Самое простое обобщение уравнений ДЛ добавить эту силу к силе Лоренца. Вторая формулировка модели ДЛ опирается на систему уравнений аномальной динамики электронов в кристалле. Результирую- 
щее уравнение квазиклассического движения электрона включает поправки, содержащие связность Берри. В приближении слабых полей оно совпадает с простейшим обобщением уравнения ДЛ. Полученное уравнение движения будет использовано для определения нелинейного отклика среды.

\section{Топологические поляризуемость и намагниченность, плотности заряда и тока}

Для топологических изоляторов ключевым свойством является наличие топологического магнитоэлектрического эффекта [13-15]. Поляризация и намагниченность в этом случае даются [16] выражениями

$$
\begin{aligned}
& \mathbf{P}_{T}=\left(n+\frac{1}{2}\right) \frac{e^{2}}{c h} \mathbf{B}, \\
& \mathbf{M}_{T}=\left(n+\frac{1}{2}\right) \frac{e^{2}}{c h} \mathbf{E},
\end{aligned}
$$

где $n$ - целое число.

Если использовать обозначение для постоянной тонкой структуры $\alpha=e^{2} / c \hbar$ и ввести обозначение для параметра $\theta=1+2 n$, то электрическую и магнитную индукцию можно представить как

$$
\mathbf{D}=\mathbf{E}+4 \pi \mathbf{P}_{c}+\theta \alpha \mathbf{B}, \quad \mathbf{H}=\mathbf{B}-4 \pi \mathbf{M}_{c}-\theta \alpha \mathbf{E} .
$$

Здесь индекс „,с, отмечает величины нетопологической природы.

Для топологического изолятора параметр $\theta$ равен нечетному числу $1+2 n$ внутри и равен нулю вне топологического изолятора. Для полуметалла Вейля предполагается, что $\theta=\left(\mathbf{b} \cdot \mathbf{r}-b_{0} t\right)$ [17]. Далее будет предполагаться, что этот параметр зависит от координат и времени $\theta(\mathbf{r}, t)$ как в [16]. В [18] $\theta(\mathbf{r}, t)$ рассматривается как аксионное поле.

В обычной электродинамике плотность связанных зарядов $\varrho_{c}$ связывается с поляризацией соотношением $\varrho_{c}=-\operatorname{div} \mathbf{P}_{c}$. Воспользовавшись формулой (1), можно найти, что

$$
\operatorname{div} \mathbf{P}_{T}=\frac{\alpha}{4 \pi} \nabla(\theta \mathbf{B})=\frac{\alpha}{4 \pi}(\nabla \theta \cdot \mathbf{B}) .
$$

Здесь учитывалось, что магнитных зарядов нет $(\operatorname{div} \mathbf{B}=0)$. Следовательно, можно определить топологическую плотность зарядов (аксионных зарядов) как

$$
\varrho_{T}=-\frac{\alpha}{4 \pi}(\nabla \theta \cdot \mathbf{B}) .
$$

Плотность тока (связанных) зарядов в обычной электродинамике определяется формулой

$$
\mathbf{j}_{c}=\frac{\partial \mathbf{P}_{c}}{\partial t}+c \operatorname{rot} \mathbf{M}_{c}
$$

Плотность полного тока в топологических материалах есть сумма $\mathbf{j}_{\text {tot }}=\mathbf{j}_{c}+\mathbf{j}_{T}$, где плотность топологического тока будет определена как

$$
\mathbf{j}_{T}=\frac{\partial \mathbf{P}_{T}}{\partial t}+c \operatorname{rot} \mathbf{M}_{T}
$$

Используя формулы (1) и (2), можно записать

$$
\mathbf{j}_{T}=\frac{\alpha c}{4 \pi}\left[\frac{1}{c} \frac{\partial \theta}{\partial t} \mathbf{B}+\theta \frac{1}{c} \frac{\partial \mathbf{B}}{\partial t}+\nabla \theta \times \mathbf{E}+\theta \operatorname{rot} \mathbf{E}\right] .
$$

Поскольку, согласно уравнениям Максвелла,

$$
\operatorname{rot} \mathbf{E}=-\frac{1}{c} \frac{\partial \mathbf{B}}{\partial t},
$$

плотность тока $\mathbf{j}_{T}$ определена выражением

$$
\mathbf{j}_{T}=\frac{\alpha c}{4 \pi}\left[\frac{1}{c} \frac{\partial \theta}{\partial t} \mathbf{B}+\nabla \theta \times \mathbf{E}\right] .
$$

Плотности зарядов и токов теперь могут быть добавлены к обычным плотностям зарядов и токов для того, чтобы получить обобщенные уравнения Максвелла:

$$
\begin{gathered}
\operatorname{rot} \mathbf{E}=-\frac{1}{c} \frac{\partial \mathbf{B}}{\partial t}, \quad \operatorname{div} \mathbf{B}=0, \\
\operatorname{rot} \mathbf{H}=\frac{1}{c} \frac{\partial \mathbf{D}}{\partial t}+\frac{4 \pi}{c} \mathbf{j}_{c}+\alpha\left(\frac{1}{c} \frac{\partial \theta}{\partial t} \mathbf{B}+\nabla \theta \times \mathbf{E}\right), \\
\operatorname{div} \mathbf{D}=4 \pi \varrho_{c}-\alpha \nabla \theta \cdot \mathbf{B} .
\end{gathered}
$$

Полученные обобщенные уравнения Максвелла обычно используются для описания электромагнитных явлений в топологических средах [19-22]. Если переопределить параметр $\theta$, записав его как $-2 \theta$, то система уравнений (5)-(7) примет форму уравнений „аксионной электродинамики“ [18].

\section{Простейшее обобщение уравнения Друде-Лоренца}

Пусть потенциал, удерживающий заряженную частицу, и взаимодействие между частицами отсутствуют. Движение частиц обусловлено только взаимодействием с внешним электромагнитным полем. Если аксионное поле статическое, $\theta(t, \mathbf{r})=\theta(\mathbf{r})$, то выражение для плотности тока упрощается:

$$
\mathbf{j}_{T}=\frac{\alpha c}{4 \pi} \nabla \theta \times \mathbf{E} .
$$

Сила, действующая на заряженную частицу, есть сила Лоренца, которая действует на обычные заряды и токи, а также на топологические заряды и их токи. Для одной частицы уравнение движения имеет вид

$$
\dot{\mathbf{p}}=e_{\mathrm{tot}} \mathbf{E}+\frac{1}{c} \mathbf{j}_{\mathrm{tot}} \times \mathbf{B},
$$


где $\mathbf{p}=m \dot{\mathbf{r}}-$ импульс. Здесь и далее точкой обозначены производные по времени. С учетом (3) и (8), следует записать

$$
e_{\mathrm{tot}}=e-\frac{\alpha}{4 \pi}(\nabla \theta \cdot \mathbf{B}), \quad \mathbf{j}_{\mathrm{tot}}=e \dot{\mathbf{r}}+\frac{\alpha c}{4 \pi} \nabla \theta \times \mathbf{E} .
$$

Подстановка этих выражений в уравнение движения дает

$\dot{\mathbf{p}}=\left(e \mathbf{E}+\frac{e}{c} \dot{\mathbf{r}} \times \mathbf{B}\right)+\frac{\alpha}{4 \pi}[-(\nabla \theta \cdot \mathbf{B}) \mathbf{E}+(\nabla \theta \times \mathbf{E}) \times \mathbf{B}]$.

Раскрыв двойное векторное произведение, это уравнение можно переписать в следующем виде:

$$
\dot{\mathbf{p}}=\left(e \mathbf{E}+\frac{e}{c} \dot{\mathbf{r}} \times \mathbf{B}\right)-\frac{\alpha}{4 \pi}(\mathbf{B} \cdot \mathbf{E}) \nabla \theta .
$$

Если в это уравнение добавить слагаемое, учитывающее релаксацию импульса как силу трения $-m \gamma \dot{\mathbf{r}}$, то получим уравнение движения свободной заряженной частицы как в модели ДЛ:

$$
m \ddot{\mathbf{r}}+m \gamma \dot{\mathbf{r}}=\left(e \mathbf{E}+\frac{e}{c} \dot{\mathbf{r}} \times \mathbf{B}\right)-\frac{\alpha}{4 \pi}(\mathbf{B} \cdot \mathbf{E}) \nabla \theta .
$$

Роль потенциала $U(\mathbf{r})$, удерживающего частицу в положении равновесия, можно учесть феноменологически, получив, таким образом, простейшее обобщение уравнения Друде-Лоренца:

$$
m \ddot{\mathbf{r}}+m \gamma \dot{\mathbf{r}}=-\nabla U(\mathbf{r})+\left(e \mathbf{E}+\frac{e}{c} \dot{\mathbf{r}} \times \mathbf{B}\right)-\frac{\alpha}{4 \pi}(\mathbf{B} \cdot \mathbf{E}) \nabla \theta .
$$

Из (10) следует хорошо известный факт, что в случае топологического изолятора заряды в объеме материала ведут себя как в обычных средах [14]. В объеме $\theta=\theta_{0}-$ постоянная величина, токи текут по поверхности топологического изолятора. Оценив градиент аксионного поля как $\nabla \theta \approx-\theta_{0} / l_{s} \mathbf{n}$, где $l_{s}-$ толщина переходного слоя и $\mathbf{n}$ - нормаль к границе топологического изолятора, можно для поверхностных зарядов записать уравнение ДЛ в форме

$$
m \ddot{\mathbf{r}}+m \gamma \dot{\mathbf{r}}=\left(e \mathbf{E}+\frac{e}{c} \dot{\mathbf{r}} \times \mathbf{B}\right)+\frac{\alpha \theta_{0}}{4 \pi l_{s}}(\mathbf{B} \cdot \mathbf{E}) \mathbf{n} .
$$

Из полученного результата видно, что учет топологических свойств материала приводит к появлению „аномального слагаемого“, которое пропорционально произведению электрического и магнитного полей. Это слагаемое наряду с силой Лоренца дает вклад в нелинейный отклик среды на внешнее электромагнитное поле.

Стоит отметить, что если взять уравнения (5)-(7) в качестве уравнений, описывающих электромагнитное поле в среде, и вычислить стандартным методом [23,24] силу, действующую на вещество в электромагнитном поле, то помимо известных сил добавится сила $\mathbf{f}_{T}=-(\alpha / 4 \pi)(\mathbf{B} \cdot \mathbf{E}) \nabla \theta$.

Дополнительное слагаемое в (12) содержит произведение полей $\mathbf{B} \cdot \mathbf{E}$. Для поперечных электромагнитных полей это произведение равно нулю. Однако вблизи границ раздела, в неоднородных средах или около источника излучения электромагнитные поля не поперечны.

\section{Аномальная динамика электронов}

Движение электронов в квазиклассическом приближении описывается системой уравнений $[5]^{1}$

$$
\dot{\mathbf{r}}=\mathbf{v}(\mathbf{p}), \quad \dot{\mathbf{p}}=e \mathbf{E}+\frac{e}{c} \dot{\mathbf{r}} \times \mathbf{B} .
$$

Первое уравнение определяет скорость, которая связана с энергией частицы как

$$
\mathbf{v}(\mathbf{p})=\frac{\partial \mathscr{E}(\mathbf{p})}{\partial \mathbf{p}}
$$

Если взять кинетическую энергию заряженной частицы в виде $\mathscr{E}(\mathbf{p})=\mathbf{p}^{2} / 2 m$, то для скорости получится выражение $\mathbf{v}=\mathbf{p} / m$. Для дираковской частицы $\mathscr{E}(\mathbf{p})=\mathbf{v}_{0} \cdot\left(\mathbf{p}-\mathbf{p}_{0}\right)$. Второе уравнение есть выражение силы Лоренца, с которой электромагнитное поле действует на электроны. Электроны предполагаются почти свободными и нет межзонных переходов.

Состояние электрона в периодическом поле кристаллической решетки описывается векторами состояния Блоха $|\psi\rangle=\exp (i \mathbf{p} \cdot \mathbf{r} / \hbar)\left|u_{\mathbf{p}}(\mathbf{r})\right\rangle$, где $\mathbf{p}-$ квазиимпульс электрона, $\left|u_{\mathbf{p}}(\mathbf{r})\right\rangle=\left|u_{\mathbf{p}}\left(\mathbf{r}+\mathbf{r}_{\text {lat }}\right)\right\rangle$ для любого вектора прямой решетки $\mathbf{r}_{\text {lat }}$. Состояния Блоха дополнительно индексируются номерами зон. Предположим, что межзонных переходов нет, тогда номер зоны можно опустить.

Динамика зонных электронов описывается системой уравнений [25-29]

$$
\begin{array}{r}
\dot{\mathbf{r}}=\mathbf{v}+\dot{\mathbf{p}} \times \Omega(\mathbf{p}), \\
\dot{\mathbf{p}}=e \mathbf{E}+\frac{e}{c} \dot{\mathbf{r}} \times \mathbf{B},
\end{array}
$$

в которых $\mathbf{r}$ - положение центра волнового пакета зонных электронов, p - вектор квазиимпульса, сопряженного координате r. Вектор $\Omega(\mathbf{p})$ - вектор кривизны Берри, компоненты которого определены соотношениями $\Omega_{i}=\varepsilon_{i j k} \partial A_{k} / \partial p_{j}$. Входящий в эту формулу вектор $\mathbf{A}(\mathbf{p})$, называемый связностью Берри, вычисляется при помощи периодических амплитуд функций Блоха:

$$
\mathbf{A}(\mathbf{p})=i\left\langle u_{\mathbf{p}}|\partial / \partial \mathbf{p}| u_{\mathbf{p}}\right\rangle \text {. }
$$

Нетривиальные свойства зон Бриллюэна могут отразиться в ненулевом значении вектора $\mathbf{A}(\mathbf{p})$. Таким образом, топологические свойства диэлектрических и проводящих сред описываются с помощью кривизны Берри $\boldsymbol{\Omega}(\mathbf{p})[12,30]$. Вектор $\boldsymbol{\Omega}(\mathbf{p})$ формально можно понимать как эффективное магнитное поле, которое определено на импульсном пространстве и которое приводит к возникновению аномальной скорости и эффекту Холла без внешнего магнитного поля [31]. Учет аномальной скорости приводит к системе уравнений (13) и (14).

\footnotetext{
${ }^{1}$ Обычно используется вектор квазиимпульса $\mathbf{k}$, вместо которого здесь берется $\mathbf{p}=\hbar \mathbf{k}$.
} 
Из уравнений (13) и (14) можно выразить $\dot{\mathbf{p}}[26,29]$ :

$$
\frac{m^{*}}{m} \dot{\mathbf{p}}=\left(e \mathbf{E}+\frac{e}{c} \mathbf{v} \times \mathbf{B}\right)+\frac{e^{2}}{c}(\mathbf{E} \cdot \mathbf{B}) \boldsymbol{\Omega},
$$

где эффективная масса $m^{*}$ определена формулой

$$
m^{*}=m\left[1+\frac{e}{c}(\boldsymbol{\Omega} \cdot \mathbf{B})\right] .
$$

Если рассмотреть топологический изолятор, когда плотность тока дается выражением (8), и записать $\dot{\mathbf{r}}=\mathbf{v}+e \mathbf{E} \times \Omega$, то для аномального тока (связанного с аномальной скоростью в (13)) получится выражение $\mathbf{j}_{T}=-e^{2} \boldsymbol{\Omega} \times \mathbf{E}$. Сравнив это выражение с $(8)$, можно связать кривизну Берри (вектор $\boldsymbol{\Omega}$ ) с аксионным полем $\theta$ :

$$
\mathbf{\Omega}=-\frac{\alpha c}{4 \pi e^{2}} \nabla \theta=-\frac{1}{4 \pi \hbar} \nabla \theta .
$$

В приближении $m^{*}=m$

$$
\dot{\mathbf{p}}=\left(e \mathbf{E}+\frac{e}{c} \mathbf{v} \times \mathbf{B}\right)-\frac{\alpha}{4 \pi}(\mathbf{B} \cdot \mathbf{E}) \nabla \theta .
$$

Положив дополнительно $\mathbf{p}=m \mathbf{v}, \mathbf{v}=\dot{\mathbf{r}}$, можно получить уравнение (9). Вводя дополнительно учитывающее диссипацию слагаемое $-m \gamma \dot{\mathbf{r}}$, можно прийти к полученному ранее простейшему обобщенному уравнению ДЛ (10).

\section{Обобщение уравнения Друде-Лоренца}

Чтобы получить поправки к уравнению ДЛ (10), следует исходить из системы уравнений (13) и (14). Если зависимость энергии от (квази)импульса выбрана в форме $\mathscr{E}(p)=\mathbf{p}^{2} / m$ и добавлено слагаемое, описывающее релаксацию импульса, то система уравнений (13) и (14) принимает вид

$$
\begin{gathered}
\dot{\mathbf{r}}=\frac{1}{m} \mathbf{p}+\dot{\mathbf{p}} \times \boldsymbol{\Omega}(\mathbf{p}), \\
\dot{\mathbf{p}}+\gamma \mathbf{p}=e \mathbf{E}+\frac{e}{c} \dot{\mathbf{r}} \times \mathbf{B},
\end{gathered}
$$

Определив новую переменную

$$
\mathbf{R}=\mathbf{r}-\mathbf{p} \times \Omega,
$$

систему уравнений (19) и (20) можно записать в следующей форме:

$$
\begin{gathered}
m \dot{\mathbf{R}}=\mathbf{p}, \\
m \ddot{\mathbf{R}}+\gamma m \dot{\mathbf{R}}=e \mathbf{E}+\frac{e}{c} \dot{\mathbf{R}} \times \mathbf{B}+\frac{e}{c}(\dot{\mathbf{p}} \times \mathbf{\Omega}) \times \mathbf{B} .
\end{gathered}
$$

Последнее слагаемое в (22) можно переписать как

$$
(\dot{\mathbf{p}} \times \mathbf{\Omega}) \times \mathbf{B}=(\dot{\mathbf{p}} \cdot \mathbf{B}) \boldsymbol{\Omega}-(\mathbf{B} \cdot \boldsymbol{\Omega}) \dot{\mathbf{p}} .
$$

С учетом (21) можно записать

$$
(\dot{\mathbf{p}} \cdot \mathbf{B})=e(\mathbf{E} \cdot \mathbf{B})-\gamma m(\dot{\mathbf{R}} \cdot \mathbf{B}) .
$$

Подстановка этого выражения в (22) приводит к уравнению движения заряженной частицы, описываемой координатой $\mathbf{R}$ :

$$
\begin{array}{r}
m\left[1+\frac{e}{c}(\mathbf{B} \cdot \boldsymbol{\Omega})\right] \ddot{\mathbf{R}}+\gamma m\left[\dot{\mathbf{R}}+\frac{e}{c} \boldsymbol{\Omega}(\dot{\mathbf{R}} \cdot \mathbf{B})\right]= \\
=e \mathbf{E}+\frac{e}{c} \dot{\mathbf{R}} \times \mathbf{B}+\frac{e^{2}}{c}(\mathbf{E} \cdot \mathbf{B}) \boldsymbol{\Omega}
\end{array}
$$

Это уравнение можно понимать как обобщение уравнения движения заряженной частицы в электромагнитном поле с учетом аномальной скорости. Коэффициент перед второй производной от вектора $\mathbf{R}$ есть эффективная масса. Релаксационное слагаемое приобрело поправку, пропорциональную $\Omega$ и напряженности магнитного поля.

Если считать, что магнитное поле невелико, так что $(e / c) B \Omega \ll 1$, то можно пренебречь топологическими поправками в эффективной массе и в релаксационном коэффициенте. Уравнение (23) тогда примет следующий вид:

$$
m \ddot{\mathbf{R}}+\gamma m \dot{\mathbf{R}}=e \mathbf{E}+\frac{e}{c} \dot{\mathbf{R}} \times \mathbf{B}+\frac{e^{2}}{c}(\mathbf{E} \cdot \mathbf{B}) \boldsymbol{\Omega},
$$

что есть обобщенное уравнение ДЛ (10), в котором координата $\mathbf{r}$ заменена на „удлиненную“ координату $\mathbf{R}$.

Существенную роль играл выбор зависимости энергии от квазиимпульса $\mathscr{E}(p)=\mathbf{p}^{2} / m$. Для зависимости „релятивистского“ типа уравнение движения будет иным. Зависимость кривизны Берри $\boldsymbol{\Omega}(\mathbf{p})$ от квазиимпульса может быть дополнительной причиной нелинейного отклика среды. Но далее будет предполагаться, что $\Omega$ есть постоянный вектор.

\section{Определение токов}

Если рассматривать поверхностный ток на границе топологического изолятора, моделируя его свободными зарядами, то расчет нелинейного отклика можно провести на основе обобщенной модели ДЛ, описываемой уравнением (18). Релаксацию импульса можно учесть, добавив слагаемое, которое обеспечивает затухание тока в отсутствии внешних сил. Соответствующее уравнение получено в предыдущем разделе (24). Введя переменную $\mathbf{P}=m \dot{\mathbf{R}}$, уравнению (24) можно придать следующий вид:

$$
\dot{\mathbf{P}}+\gamma \mathbf{P}=\left(e \mathbf{E}+\frac{e}{c m} \mathbf{P} \times \mathbf{B}\right)+\frac{e^{2}}{c}(\mathbf{E} \cdot \mathbf{B}) \boldsymbol{\Omega} .
$$

Следуя рецепту из [9], $\mathbf{P}$ представляется рядом $\mathbf{P}=\mathbf{P}^{(0)}+c^{-1} \mathbf{P}^{(1)}+c^{-2} \mathbf{P}^{(2)}+\ldots$, который подставляется в (25). Собирая слагаемые перед одинаковыми степенями $c^{-1}$, можно получить систему уравнений

$$
\begin{gathered}
\dot{\mathbf{P}}^{(0)}+\gamma \mathbf{P}^{(0)}=e \mathbf{E}, \\
\dot{\mathbf{P}}^{(1)}+\gamma \mathbf{P}^{(1)}=\frac{e}{m} \mathbf{P}^{(0)} \times \mathbf{B}+e^{2}(\mathbf{E} \cdot \mathbf{B}) \boldsymbol{\Omega},
\end{gathered}
$$




$$
\dot{\mathbf{P}}^{(2)}+\gamma \mathbf{P}^{(2)}=\frac{e}{m} \mathbf{P}^{(1)} \times \mathbf{B}
$$

и т. д. Для гармонических полей

$$
\mathbf{E}=\mathbf{E}_{0} e^{-i \omega_{c} t}+\text { c.c. }, \quad \mathbf{B}=\mathbf{B}_{0} e^{-i \omega_{c} t}+\text { c.c. },
$$

фурье-компонента $\mathbf{P}^{(0)}(\omega)$ определяется из (26):

$$
\mathbf{P}^{(0)}(\omega)=\frac{e \mathbf{E}_{0}}{\gamma-i \omega} 2 \pi \delta\left(\omega-\omega_{c}\right)+\frac{e \mathbf{E}_{0}^{*}}{\gamma+i \omega} 2 \pi \delta\left(\omega+\omega_{c}\right) .
$$

Отсюда

$$
\mathbf{P}^{(0)}(t)=\frac{e \mathbf{E}_{0}}{\gamma-i \omega_{c}} e^{-i \omega_{c} t}+\frac{e \mathbf{E}_{0}^{*}}{\gamma+i \omega_{c}} e^{i \omega_{c} t} .
$$

Подстановка (29) в (27) приводит к уравнению

$$
\dot{\mathbf{P}}^{(1)}+\gamma \mathbf{P}^{(1)}=\mathbf{F}_{0} e^{-i 2 \omega_{c} t}+\mathbf{F}_{0}^{*} e^{i 2 \omega_{c} t}+\mathbf{G}_{0},
$$

где

$$
\begin{gathered}
\mathbf{F}_{0}=\frac{e^{2} \mathbf{E}_{0} \times \mathbf{B}_{0}}{m\left(\gamma-i \omega_{c}\right)}+e^{2}\left(\mathbf{E}_{0} \cdot \mathbf{B}_{0}\right) \boldsymbol{\Omega} \\
\mathbf{G}_{0}=\frac{e^{2} \mathbf{E}_{0} \times \mathbf{B}_{0}^{*}}{m\left(\gamma-i \omega_{c}\right)}+\frac{e^{2} \mathbf{E}_{0}^{*} \times \mathbf{B}_{0}}{m\left(\gamma+i \omega_{c}\right)}+2 e^{2} \operatorname{Re}\left(\mathbf{E}_{0} \cdot \mathbf{B}_{0}^{*}\right) \boldsymbol{\Omega} .
\end{gathered}
$$

Решение уравнения (30) стандартным образом приводит к выражению

$$
\mathbf{P}^{(1)}(t)=\frac{\mathbf{F}_{0}}{\gamma-i 2 \omega_{c}} e^{-i 2 \omega_{c} t}+\frac{\mathbf{F}_{0}^{*}}{\gamma+i 2 \omega_{c}} e^{i 2 \omega_{c} t}+\gamma^{-1} \mathbf{G}_{0} .
$$

Первые слагаемые в (31) описывают генерацию второй гармоники, последнее слагаемое - оптическое выпрямление. Процедуру можно продолжать и получить вклады в $\mathbf{P}(t)$ более высоких порядков по амплитудам полей.

Пусть плотность тока определяется формулой $\mathbf{j}=e n_{e} \dot{\mathbf{r}}$, где $n_{e}-$ плотность носителей тока. В этом случае выражение для плотности тока имеет вид

$$
\mathbf{j}=\frac{e n_{e}}{m}(\mathbf{P}+m \dot{\mathbf{P}} \times \mathbf{\Omega})
$$

Поскольку $\mathbf{P}(t)$ представлено рядом по степеням $c^{-1}$, плотность тока есть такой же ряд. Для линейного тока $\mathbf{j}^{(0)}$ из полученных результатов следует выражение

$$
\begin{aligned}
\mathbf{j}^{(0)}(t)= & \frac{e^{2} n_{e}}{m\left(\gamma-i \omega_{c}\right)} \mathbf{E}_{0} e^{-i \omega_{c} t} \\
& -\frac{i \omega_{c} e^{2} n_{e}}{\left(\gamma-i \omega_{c}\right)} \mathbf{E}_{0} \times \Omega e^{-i \omega_{c} t}+c . c .
\end{aligned}
$$

Если определить линейную проводимость формулой

$$
\sigma(\omega)=\frac{e^{2} n_{e}}{m\left(\gamma-i \omega_{c}\right)},
$$

то выражение для линейного тока примет следующий вид

$$
\mathbf{j}^{(0)}(t)=\sigma\left(\omega_{c}\right) \mathbf{E}_{0} e^{-i \omega_{c} t}+i m \omega_{c} \sigma\left(\omega_{c}\right) \boldsymbol{\Omega} \times \mathbf{E}_{0} e^{-i \omega_{c} t}+c . c .
$$

Подставив (31) в

$$
\mathbf{j}^{(1)}=\frac{e n_{e}}{m}\left(\mathbf{P}^{(1)}+m \dot{\mathbf{P}}^{(1)} \times \mathbf{\Omega}\right),
$$

можно получить плотность тока, отвечающую за нелинейные эффекты второго порядка - генерацию второй гармоники и оптическое выпрямление. Пусть компоненты тензоров нелинейных проводимостей вводятся следующими соотношениями:

$$
\begin{aligned}
j_{j}^{(1)}\left(2 \omega_{c}\right)= & \sigma_{j k l}^{(2)}\left(2 \omega_{c} ; \omega_{c}, \omega_{c}\right) E_{0 k} B_{0 l}, \\
j_{j}^{(1)}(0)= & \sigma_{j k l}^{(2)}\left(0 ; \omega_{c},-\omega_{c}\right) E_{0 k} B_{0 l}^{*} \\
& +\sigma_{j k l}^{(2)}\left(0 ;-\omega_{c}, \omega_{c}\right) E_{0 k}^{*} B_{0 l} .
\end{aligned}
$$

Тензор нелинейной проводимости $\sigma_{j k l}^{(2)}$ представляется суммой обычной $\hat{\sigma}_{j k l}^{(2)}$ и аномальной (топологической) $\tilde{\sigma}_{j k l}^{(2)}$ частей. Вычисления на основе полученных результатов приводят к следующим выражениям:

$$
\begin{gathered}
\hat{\sigma}_{j k l}^{(2)}(2 \omega ; \omega, \omega)=\frac{n_{e} e^{3} \varepsilon_{j k l}}{m^{2} c(\gamma-2 i \omega)(\gamma-i \omega)}, \\
\hat{\sigma}_{j k l}^{(2)}\left(0 ; \omega_{c},-\omega_{c}\right)=\frac{e^{3} n_{e}}{m^{2} c \gamma(\gamma-i \omega)} \varepsilon_{j k l} .
\end{gathered}
$$

Для аномальной части нелинейной проводимости $\tilde{\sigma}_{j k l}^{(2)}\left(2 \omega_{c} ; \omega_{c}, \omega_{c}\right)$ можно получить следующее выражение:

$$
\tilde{\sigma}_{j k l}^{(2)}\left(2 \omega_{c} ; \omega_{c}, \omega_{c}\right)=\frac{e^{3} n_{e} \Omega_{j} \delta_{k l}}{\gamma-2 i \omega_{c}}+\frac{2 i \omega_{c} e^{3} n_{e} \Omega_{m} \varepsilon_{j m n} \varepsilon_{n k l}}{c\left(\gamma-2 i \omega_{c}\right)\left(\gamma-i \omega_{c}\right)} .
$$

Используя выражение для линейной проводимости $\sigma(\omega),(33)$ можно переписать в виде

$$
\begin{aligned}
\tilde{\sigma}_{j k l}^{(2)}\left(2 \omega_{c} ; \omega_{c}, \omega_{c}\right)= & \frac{e}{c} \sigma\left(2 \omega_{c}\right) \Omega_{j} \delta_{k l} \\
& +2 i \frac{\omega_{c} m}{c e n_{e}} \sigma\left(\omega_{c}\right) \sigma\left(2 \omega_{c}\right) \Omega_{m} \varepsilon_{j m n} \varepsilon_{n k l} .
\end{aligned}
$$

Первое слагаемое в этом выражении симметричное по двум последним индексам, т.е. это диагональный элемент тензоров $\left(\tilde{\sigma}_{j}^{(2)}\right)$. Второе слагаемое, напротив, антисимметричное и есть недиагональный элемент $\left(\tilde{\sigma}_{j}^{(2)}\right)$.

Рассматривая топологические изоляторы, можно воспользоваться формулой (17) и представить нелинейную проводимость (33) в виде

$$
\begin{gathered}
\tilde{\sigma}_{j k l}^{(2)}\left(2 \omega_{c} ; \omega_{c}, \omega_{c}\right)=\frac{\alpha}{4 \pi e} \sigma\left(2 \omega_{c}\right) b_{j} \delta_{k l} \\
+\frac{2 i \omega_{c} m \alpha}{4 \pi e^{3} n_{e}} \sigma\left(\omega_{c}\right) \sigma\left(2 \omega_{c}\right) b_{m} \varepsilon_{j m n} \varepsilon_{n k l},
\end{gathered}
$$

где $\mathbf{b}=-\nabla \theta$. Отсюда следует, что для диагональной части нелинейной проводимости справедливо соотношение

$$
\tilde{\sigma}_{j l l}^{(2)}(2 \omega ; \omega, \omega)=\frac{\alpha}{4 \pi e} \sigma(2 \omega) b_{j} .
$$


Замечательно, что в подобного рода соотношениях (соотношения Миллера [11]) детали модели обычно исчезают.

Для случая топологического изолятора можно использовать следующее приближение: $\mathbf{b}=-\nabla \theta \approx \theta_{0} / l_{s}, l_{s}-$ толщина слоя, на котором поле $\theta(r)$ спадает от $\theta_{0}$ до нуля. Таким образом, нелинейная проводимость $\tilde{\sigma}_{j k l}^{(2)}$ отлична от нуля только на поверхности изолятора и пропорциональна целым числам $\theta=1+2 n$.

\section{Заключение}

Здесь рассмотрено обобщение модели ДЛ, которое применительно к топологическим средам позволяет оценить вклады топологических характеристик среды в нелинейные оптические эффекты. Наиболее простое обобщение заключается в добавке к обычной силе Лоренца силы взаимодействия электромагнитного поля с зарядами и токами, которые отвечают за топологический магнитоэлектрический эффект. Следующим шагом на пути обобщения модели ДЛ является замена массы на эффективную массу, которая зависит от топологических свойств зонных электронов.

Для примера применения развитого в работе формализма получены выражения для нелинейных проводимостей, отвечающих за генерацию второй гармоники и оптическое выпрямление. Для случая генерации второй гармоники показано, что нелинейная проводимость пропорциональна линейной проводимости, а коэффициент пропорциональности равен постоянной тонкой структуры, умноженной на одну из компонент кривизны Берри. В случае топологического изолятора эта компонента пропорциональна целому нечетному числу.

Важно отметить, что в случае топологического изолятора, поскольку $\mathbf{b}=-\nabla \theta(\mathbf{r})$, топологическая нелинейная проводимость отлична от нуля на поверхности изолятора, но выражается через объемную линейную проводимость. Это новый пример известного для топологических теорий поля и теории топологических сред „соответствия объем-граница“(the bulk-boundary correspondence) [32-35].

Предположение относительно квадратичной зависимости энергии от квазиимпульса было существенным при выводе уравнения (23). В случае, если $\mathscr{E}(p) \sim|\mathbf{p}|$ или неквадратичной зависимости, например $\mathscr{E}(p)=\mathbf{p}^{2} / m+g \mathbf{p}^{4}$, получить уравнение движения типа ДЛ вряд ли удастся. Однако для определения нелинейных проводимостей или восприимчивостей можно использовать систему уравнений (13) и (14) непосредственно.

При проведенном в настоящей статье расчете нелинейных проводимостей не учитывались топологические поправки к эффективной массе. Соответствующее обобщение может быть сделано путем разложения всех слагаемых в уравнении (23) по степеням $c^{-1}$ и группировкой слагаемых одного порядка по $c^{-1}$.

\section{Финансирование работы}

Исследование выполнено при поддержке Российского фонда фундаментальных исследований (грант № 18-0200921).

\section{Список литературы}

[1] Ахманов С.А., Никитин С.Ю. Физическая оптика. МГУ, 1998.

[2] Блохинцев Д.И. Основы квантовой механики. М.: Наука, 1976.

[3] Новотный Л., Хехт Б. Основы нанооптики. М.: ФИЗМАТЛИТ. 2009.

[4] Майер C. Плазмоника. Теория и приложения. М.-Ижевск: НИЦ „Регулярная и хаотическая динамика“, 2001.

[5] Киттель Ч. Квантовая теория твердых тел. М.: Наука, 1987.

[6] Xiao Di, Shi Junren, Niu Qian // Phys. Rev. Lett. 2005. V. 95. P. 137204.

[7] Ryuichi Shindou, Ken-Ichiro Imura // Nucl. Phys. B. 2005. V. 720 [FS]. P. 399-435.

[8] Watson A.B., Jianfeng Lu, Weinstein M.I. // J. Math. Phys. 2017. V. 58. P. 021503.

[9] Бломберген Н. Нелинейная оптика. М.: Мир, 1966.

[10] Шен И.Р. Принципы нелинейной оптики. М.: Наука, 1989.

[11] Ярив А. Квантовая электроника и нелинейная оптика. М.: Сов. Радио, 1973.

[12] Shapere A., Wilczek F. Geometric Phases in Physics. Singapore, Teaneck, N.J.: World Scientific, 1989.

[13] Essin A.M., Moore J.E., Vanderbilt D. Phys. Rev. Lett. 2009. V. 102 , P. 146805.

[14] Hasan M.Z., Kane C.L. // Rev. Mod. Phys. 2011. V. 83. N 4. P. 3045-3067.

[15] Xiao-Liang Qi, Shou-Cheng Zhang // Rev. Mod. Phys. 2011. V. 83. N 4. P. 1057-1110.

[16] Xiao-Liang Qi, Hughes T.L., Shou-Cheng Zhang // Phys. Rev. B. 2008. V. 78. P. 195424 [43 pages].

[17] Zyuzin A.A., Burkov A.A. // Phys. Rev. B. 2012. V. 86. P. 115133.

[18] Wilczek F. // Phys. Rev. Lett. 1987. V. 58. N 18. P 1799-1802.

[19] Goswami P., Tewari S. // Phys. Rev. B. 2013. V. 88. P. 245107.

[20] Visinelli L. Axion-Electromagnetic Waves, arXiv:1401.0709 [physics.class-ph]

[21] Zebin Qiu, Gaoqing Cao, Xu-Guang Huang // Phys. Rev. D. 2017. V. 95. P. 036002.

[22] Murchikova E.M. // J. Phys. A: Math. Theor. 2011. V. 44. P. 045401.

[23] Гинзбург В.Л., Угаров В.А. // УФН. 1976. Т. 118. № 1. C. $175-188$

[24] Макаров В.П., Рухадзе А.А. // УФН. Т. 179. № 9. C. 995-1001.

[25] Son D.T., Yamamoto N. // Phys. Rev. Lett. 2012. V. 109. P. 181602.

[26] Son D.T., Spivak B.Z. // Phys. Rev. B. 2013. V. 88. P. 104412.

[27] Son D.T., Yamamoto N. // Phys. Rev. D. 2013. V. 87. P. 085016.

[28] Imran M., Hershfield S. Phys. Rev. B. 2018. V. 98. P. 205139.

[29] Stone M., Dwivedi V. // Phys. Rev. D. 2013. V. 88. P. 045012.

[30] Di Xiao, Ming-Che Chang, Qian Niu // Rev. Mod. Phys. 2010. V. 82. N 3. P. 1959-2007. 
[31] Nagaosa N., Simova J., Onoda Sh., MacDonald A.H., Ong N.P. // Rev. Mod. Phys. 2010. V. 82. N 2. P. 1539-1592.

[32] Essin A.M., Gurarie V. // Phys. Rev. B. 2011. V. 84. P. 125132 [10 pages].

[33] Mong R.S.K., Shivamoggi V. // Phys. Rev. B. 2011. V. 83. P. 125109 [15 pages].

[34] Chenijie Wang, Chien-Hung Lin, Michael Levin M. // Phys. Rev. X. 2016. V. 6. P. 021015 (2016) [31 pages].

[35] Liang Kong, Xiao-Gang Wen, Hao Zheng // Nuck. Phys. B. 2017. V. 922. P. $62-76$. 